\title{
Estimating additional root cohesion by exploiting a root topological model based on Leonardo's Rule
}

\author{
Leonardo Valerio Noto ${ }^{1}$, Antonio Francipane ${ }^{1}$, Federico Preti ${ }^{3}$, Marco Petti ${ }^{2}$, and Elisa Arnone $^{2}$ \\ ${ }^{1}$ University of Palermo, Department of Engineering, Palermo, Italy (leonardo.noto@unipa.it) \\ ${ }^{2}$ University of Udine, DPIA, Udine, Italy \\ ${ }^{3}$ Università of Firenze, DAGRI, Firenze, Italy
}

Root topological models are schematic representations of the root structure based on a defined topology graph theory. In the context of hillslope stabilization modeling against rainfall-induced shallow landslides, the root topological models may be used in combination with root strength models assessment, such as the Root Bundle Model (RBM), to estimate the ultimate root reinforcement. The effect of plant roots on slope stabilization is determined by the interaction between soil and the hydrological processes (within the root zone) and the biotechnical characteristics of the root system, such as root length, root density, root tensile strength, root area, root diameter profile and the total number of roots. Describing adequately the root architecture of a plant species is useful, for example, to evaluate how the root structure may change in different soil and/or climatological conditions and, ultimately, as an example, to assess the most suitable plant species to be adopted.

This study exploits the potentiality of a root topological model based on Leonardo's rule in describing root architectures of (i) different species (and tree individual) at given growth conditions, (ii) same species at different environmental conditions, e.g., exposure to light, water and nutrient availability. The former is supported by field campaign measurements from Tuscany region, the latter are reproduced starting from a reference case and imposing growth assumptions. Next, the information of the root system, in terms of root length, density, root diameter profile, total number of roots, are used to estimate, through a RBM approach, the additional root tensile force, deriving it from the force-deformation theory of linear elasticity in a rigorous framework aimed to derive the additional shear resistance from the Mohr-Coulomb's failure plane.

The preliminary results demonstrated the capability of the root topological model of reproducing different types of root system; additional data are required to further validate the model, with regard to the growth conditions simulation. Similarly, laboratory test of root strength would allow to quantify the improvement derived from the rigorous method adopted to estimate the additional root strength. 